Article

\title{
Jump Aggregation, Volatility Prediction, and Nonlinear Estimation of Banks' Sustainability Risk
}

\author{
Zhouwei Wang ${ }^{1}$, Qicheng Zhao ${ }^{1}$, Min Zhu ${ }^{1}$ and Tao Pang ${ }^{2, *}$ (D) \\ 1 School of Finance and Business, Shanghai Normal University, Shanghai 200234, China; \\ wangzhouw@163.com (Z.W.); zhaoqc183@163.com (Q.Z.); zhum@shnu.edu.cn (M.Z.) \\ 2 Department of Mathematics, North Carolina State University, Raleigh, NC 27695-8205, USA \\ * Correspondence: tpang@ncsu.edu; Tel.: +1-919-513-2110
}

Received: 20 September 2020; Accepted: 21 October 2020; Published: 25 October 2020

\begin{abstract}
Extreme financial events usually lead to sharp jumps in stock prices and volatilities. In addition, jump clustering and stock price correlations contribute to the risk amplification acceleration mechanism during the crisis. In this paper, four Jump-GARCH models are used to forecast the jump diffusion volatility, which is used as the risk factor. The linear and asymmetric nonlinear effects are considered, and the value at risk of banks is estimated by support vector quantile regression. There are three main findings. First, in terms of the volatility process of bank stock price, the Jump Diffusion GARCH model is better than the Continuous Diffusion GARCH model, and the discrete jump volatility is significant. Secondly, due to the difference of the sensitivity of abnormal information shock, the jump behavior of bank stock price is heterogeneous. Moreover, CJ-GARCH models are suitable for most banks, while ARJI-R2-GARCH models are more suitable for small and medium sized banks. Thirdly, based on the jump diffusion volatility information, the performance of the support vector quantile regression is better than that of the parametric quantile regression and nonparametric quantile regression.
\end{abstract}

Keywords: Jump-GARCH model; jump diffusion volatility; support vector quantile regression; value at risk

\section{Introduction}

Extreme financial events can trigger a continuous downward jump in stock markets. In June 2015 , the deleveraging of China's A-shares disaster and the "811" exchange rate reform caused the RMB exchange rate to plummet, and the Chinese stock market circuit breaker kicked in four times in the first four days of January 2016, as more than 1000 stocks plunged by $10 \%$, the maximal allowed daily drop. The global COVID-19 pandemic breakout in 2020 also caused a big sell-off in global stock markets. Even if the stimulus package was launched one after another, the U.S. stock market after the upward adjustment from 9-18 March still suffered five collapses and ruptures. During this period, circuit breakers kicked in many times in the global stock markets. In particular, on 12 March, the stock markets of 11 countries triggered the circuit breaker. The price of WTI crude oil futures for May delivery on the New York Mercantile Exchange surprisingly plummeted by $305.97 \%$ on 20 April, closing at -37.63 US dollars per barrel, which was the first time in the oil futures' trading history. These crisis events continue to remind us that we must carefully measure the financial risks caused by jumps.

Banking is the main industry of indirect financing with the largest scale of financial resource financing, and the market risk of the banking industry is very common [1]. The bottom line is to prevent systemic risks, and that requires banks to effectively monitor and control systemic and sustainability risks [2,3], which is also very important for financial security and economic stability [4]. 
Value at risk (VaR) is a well-recognized effective measure of bank risk. Its estimation methods mainly include parametric, non-parametric, simulation, and quantile regression $(\mathrm{QR})$ measurement methods. One of the core input variables of these methods is the predicted volatility. The degree of accuracy and effectiveness of volatility estimation determines the effectiveness of the VaR estimation.

At present, the commonly used methods to measure and estimate volatility are historical volatility, calculated by static average and dynamic moving average; time-varying volatility, estimated by the continuous generalized autoregressive conditional heteroscedasticity (GARCH) model [5]; implied volatility, calculated by six variable option pricing system; random volatility, generated by stochastic simulation; and realized volatility, calculated by high-frequency data. These methods all assume that the return on assets is a continuous process, and the jump component is not fully considered or is completely ignored. This assumption is not in line with the reality [6], so it cannot well explain the smile phenomenon of volatility and the Risk Amplification Acceleration Mechanism of stock price jumps and its clustering caused by extreme financial events or abnormal information shocks $[7,8]$. Empirical studies have shown that jumps are common in China's stock markets and have a significant positive impact on the volatility of China's stock markets [9-11].

The jump volatility ratio of asset price is usually around 0.44 . The jump diffusion process not only has higher pricing efficiency, but also can fit the right-skewed, peak, heavy-tail, clustering, and leverage effect characteristics of asset return distributions and can effectively explain the smile feature of volatility [12]. The jump risk factor has a high ability to improve the interpretation ability of risk prediction $[13,14]$. In addition, the influence mode of risk factors on VaR can be linear or nonlinear. Only considering the linear influence model would overlook the relatively significant nonlinear effect of risk factors and reduce the accuracy of risk estimation model $[15,16]$.

In the current literature on risk measurement of financial institutions, the limitations of those models lead to the neglect and underestimation of extreme risk events and systemic risk shocks. It may increase the opportunity cost of risk prevention and control and increase the probability of financial system failure. To overcome the issues, in this paper, we use the most effective jump volatility model to predict the volatility of bank stock price, and we take the jump volatility prediction value as one of the core input variables. Then we use the nonlinear support vector quantile regression (SVQR) technology to estimate the market VaR of the banking industry.

The contributions of this paper are as follows. Firstly, we consider the impact of abnormal information and we use the Jump-GARCH model to fit the jump volatility of bank stock price and to estimate the conditional volatility under continuous volatility and jump diffusion volatility models. Secondly, we introduce the SVQR technology to estimate conditional value at risk, which can accommodate the linear and non-linear effects of risk factors. Thirdly, we extend the VaR estimation method to be able to measure normal risk and extreme risk sensitively at the same time, which makes the risk monitoring more reasonable and realistic. It also provides a theoretical basis for the measurement of systemic financial risk spillover.

The rest of this paper is organized as follows. In Section 2, we review the related literature on jump aggregation, volatility prediction, and nonlinear estimation of banks' sustainability risk. In Section 3, we present the models and the methodology in view of the non-linear estimation of the value at risk of banks' sustainability risk. In Section 4, we conduct some empirical studies and do some further analysis. Finally, we summarize our findings and conclude the paper in Section 5.

\section{Literature Review}

\subsection{Literature Review on Volatility Prediction}

Stock price volatility is the main input variable to estimate value at risk by using the parametric method. Based on the different algorithm principles, the volatility prediction approaches can be classified into two methods, single estimation method and combination estimation method [17]. The former is mainly static average, moving average, and GARCH. The latter mainly uses the option 
pricing formula to calculate the implied volatility of bank stock price return. Among them, the single variable GARCH model can fit the characteristics of right deviation, peak, heavy tail, clustering, and asymmetry, and can estimate the time-varying volatility. Therefore, it has become the mainstream volatility prediction approach. An autoregressive conditional heteroscedasticity (ARCH) model is proposed at first, and it is extended to the generalized ARCH (GARCH) model. In order to fit the volatility asymmetry caused by external shocks, exponential GARCH (EGARCH) and fractionally integrated GARCH (FIGARCH) are developed. Many empirical comparative studies show that the EGARCH model can provide the most effective volatility prediction among the single variable GARCH models [18].

With the development of electronic trading, high-frequency data has become the main data source of volatility prediction. With high-frequency data analysis, some researchers use realized variance to measure volatility and find that the change of financial asset return has a continuous volatility component, but also has a jump volatility component, volatility clustering, leverage effect, long memory, and volatility smile phenomenon. However, the classical GARCH model cannot describe these phenomena well. Some researchers add discrete jump into the mean value equation to improve the model performance. In particular, Reference [19] proposed the heteroscedasticity autoregressive (HAR) Jump-GARCH model by using the short memory hypothesis with lag cascading structure to approximate the long memory process. Moreover, Reference [20] introduced leverage effect to propose the HAR Jump-GARCH model with leverage effect. In addition, the realized HAR Jump-GARCH model after the expansion of the wave rate dynamic equation can capture the long memory feature [21].

We believe that the Jump-GARCH model has a good performance in volatility prediction. In this paper, we focus on different type of Jump-GARCH models, and our results confirm the good performance in volatility prediction.

\subsection{Literature Review on Bank VaR Estimation}

The most commonly used index to measure the market risk of banks is VaR. There are three kinds of estimation methods: parametric method, nonparametric method, and semi parametric method.

Parametric method mainly uses a GARCH model with normal distribution, t-distribution, and extreme value theory (EVT) distribution to estimate volatility, construct yield distribution, and estimate the VaR of large banks in the US [22]. Kupiec and Christoffersen tests show that a GARCH $(1,1)$ model with random term subject to Student's $t$ distribution has the best fitting effect on arch effect [23]. Skewed $t$ distribution can be used to estimate VaR, and it can effectively measure extreme risk [24].

Nonparametric methods mainly include historical simulations [25] and Monte Carlo simulations. The semi parametric method mainly includes extreme value theory (EVT) [26] and the quantile regression $(\mathrm{QR})$ estimation method, while the $\mathrm{QR}$ estimation method includes the linear $\mathrm{QR}$ estimation method and nonlinear QR estimation method. Each method has its advantages and disadvantages in risk reduction. Among them, the parameter QR estimation method estimates the VaR based on the prior theory of the rate of return distribution and the estimated values of its distribution parameters (mainly mean value, variance, etc.). The parametric $Q R$ method can obtain analytical solutions and has high efficiency in estimation and prediction, so it has become the first choice of VaR estimation. However, the distribution of return on financial assets is complex, so it is difficult to fit it with one kind of distribution, which makes the prediction accuracy not very ideal. In a statistical sense, VaR is the quantile corresponding to the confidence level in the risk loss distribution, while QR estimation directly estimates the specific quantile based on the empirical distribution. Therefore, the linear $Q R$ method was used to estimate the VaR.

As the action path and mode of risk caused by risk factors were complex and nonlinear $[27,28]$, we first proposed a conditional autoregressive quantile regression estimation model (CAViaR) and directly constructed four quantile evolution path equations. It was discovered that the IGARCH-CAViaR model is more suitable for describing the evolution process of financial market risk in the relatively 
mature United States and Japan, while the QR-GARCH model is more suitable for describing the evolution process of financial market risk and has performed well in emerging financial markets, especially in estimating the $99 \% \mathrm{VaR}$ of market index returns. In order to improve the estimation effect, Reference [29] proposed the neural network quantile regression technology. Reference [15] combined support vector machine technology with QR technology, and it was found that its quantile estimation effect was better than the linear parameter QR method [30].

In this paper, we combine the Jump-GARCH model and support vector QR (SVQR) estimation technology together to get an effective VaR forecast.

\section{Jump Diffusion Volatility Prediction Model and Nonlinear Estimation of VaR}

\subsection{Stock Price Jump Identification}

For a sufficiently small time interval $\delta$, assume that, within the period $[t, t+\delta]$, the time-varying threshold is given by $\vartheta_{t}(\delta)=\bar{\vartheta} h_{t}$, where $h_{t}$ is the conditional standard deviation of the rate of return. We set the adjustable parameter $\bar{\vartheta}=9$. Then, when the mean value of the first-order difference $\Delta R_{t}$ of the yield is greater than three times its standard deviation, the probability $p$ is used to judge the jump in the interval [31,32]. The conditional standard deviation is estimated by the model shown as the following:

$$
\begin{gathered}
\Delta R_{t}=R_{t}-R_{t-1}=\mu+\varepsilon_{t} \\
\varepsilon_{t}=z_{t} \sqrt{h_{t}}, z_{t} \sim N(0,1) \\
h_{t}=\omega+\alpha \varepsilon_{t-1}^{2}+\beta h_{t-1}
\end{gathered}
$$

\subsection{Selection of Jump Diffusion Volatility Prediction Model}

Stock price volatility not only has the classic characteristics of right-skewed, peak, heavy-tail, clustering, asymmetry, and long memory [33], but also has jump volatility and the volatility smile phenomenon [34,35]. The realized volatility includes a continuous volatility component and a jump volatility component. The jump volatility component has a significant positive impact on the daily, weekly, and monthly realized volatility prediction of China's stock market [36].

Volatility forecasting methods mainly include classical methods (e.g., moving average forecasting method, time series forecasting method, and $\mathrm{ARCH}$ forecasting method), basic $\mathrm{GARCH}$ family models, and recent developed GARCH extension models. The GARCH expansion models include jump GARCH models, mixing GARCH models, and long memory models (e.g., high frequency ARFIMA models, low frequency FIGARCH models, and FIEGARCH models).

Even for the classical characteristics of stock price volatility, the classical continuous diffusion volatility prediction method cannot fully describe and explain all the phenomena. The basic GARCH models can describe the right-skewed, peak, heavy-tail, clustering, and asymmetry characteristics of stock price volatility, but the structure of the model is too simple to describe the long memory, jump volatility, and volatility smile phenomena, and the short-term prediction error is often large. Long-term memory model can only describe the long-term memory. High frequency prediction models of heterogeneous autoregressive (HAR) volatility and low frequency prediction models are suitable for short-term volatility prediction, and the mixing frequency GARCH model is better than other models in predicting medium and long-term volatilities $[37,38]$, but it mainly focuses on the identification of volatility-influencing factors and simplified description of their action mechanisms. In addition, the heterogeneous autoregressive realized volatility model (HAR family model) proposed by [19] is a simple volatility long memory model. The estimation method of this kind of model is simple, and the out-of-the-sample prediction results are also good. The realized volatility (RV) HAR jump (HAR-RV-V) model captures the long memory part of realized volatility in a very simple mode. The further developed HAR-RV-CJ model considers the jump volatility component. However, the HAR family models only use the lag term to reflect the clustering, long memory and jump characteristics. 
It belongs to a simple single equation model, and it cannot describe the process and characteristics of stock price fluctuation comprehensively. In this paper, we use the Jump-GARCH model to do the forward iterative multi-step prediction method [39].

\subsection{Jump-GARCH Model}

The Jump-GARCH model adds jump terms into the mean value equation of the continuous GARCH model. The general model is as follows:

$$
\begin{gathered}
r_{t}=\mu+\sum_{i=1}^{l} \phi_{i} r_{t-i}+\sqrt{h_{t}} z_{t}+\sum_{k=1}^{n_{t}} \Upsilon_{t, k} \\
h_{t}=\omega+\sum_{i=1}^{q} \alpha_{i} \varepsilon_{t-i}^{2}+\sum_{i=1}^{p} \beta_{i} h_{t-i}
\end{gathered}
$$

Equation (4) is the mean value equation, where $r_{t}$ represents the stock return at time $t, \mu$ is a constant, $\phi_{i}$ represents the autoregressive coefficient of the stock return rate and its $i$ lag term, $l$ represents the lag order of the autoregressive equation, $h_{t}$ represents the conditional variance of the return rate, and $z_{t}$ represents the standardized disturbance term, which is subject to the standard normal distribution, $z_{t} \sim \operatorname{NID}(0,1)$. The term $\sqrt{h_{t}} z_{t}$ represents the impact of general information on the return, which is called the "continuous volatility term". $\sum_{k=1}^{n_{t}} Y_{t, k}$ is a jump term, which represents the impact of extreme events on the return, and it is called the "jump volatility term" $n_{t}$ represents the number of jumps in the return series from $t-1$ to $t$ and the jump number $n_{t}$ follows a Poisson distribution with jump intensity $\lambda_{t}\left(\lambda_{t}>0\right)$. That is, $n_{t} \sim P\left(\lambda_{t}\right)$ and the probability function is:

$$
P\left(n_{t}=j \mid \Phi_{t-1}\right)=\frac{e^{-\lambda_{i}} \lambda_{t}^{j}}{j !} \quad j=0,1,2, \cdots
$$

where $\Phi_{t}=\left[R_{t}, \cdots, R_{1}\right]$ is the information up to time $t$. In addition, $Y_{t, k}$ represents the $k$ jump amplitude of the $t$ period and $Y_{t, k} \sim N\left(\theta_{t}, \delta_{t}^{2}\right)$.

Equation (5) is the variance equation. $\omega$ is a constant, and $\varepsilon_{t-i}^{2}$ represents the square of the disturbance term of the $i$-th lag. $\alpha_{i}$ represents the coefficient of the square of the disturbance term of the $i$-th lag and $\beta_{i}$ represents the conditional variance autoregressive coefficient of the lag order.

In order to fit the different phenomena of volatility, we make different assumptions on the jump intensity, the mean value, and variance of jump amplitude for different Jump-GARCH models.

For the Constant Jump-GARCH (CJ-GARCH) model, we assume that the mean value of jump times and the mean and variance of jump amplitude are constants, that is, $\lambda_{t}=\lambda>0, \theta_{t}=\theta$, and $\delta_{t}^{2}=\delta^{2}>0$.

The ARJI-GARCH model is the Jump-GARCH model in which the jump strength is subject to an autoregressive moving average process (ARMA). For the ARJI-GARCH model, we assume that the jump strength $\lambda_{t}$ depends on the jump strength $\lambda_{t-j}$ and the jump deviation $\xi_{t-j}(j=1,2,3, \cdots)$ at the lag stage, and the jump strength $\lambda_{t}$ is subject to the autoregressive moving average process $A R M A(r, s)$ :

$$
\lambda_{t}=\lambda_{0}+\sum_{i=1}^{r} \rho_{i} \lambda_{t-i}+\sum_{i=1}^{s} \gamma_{i} \xi_{t-i}
$$

where $\lambda_{0}$ is a constant term, $\rho_{i}$ is the autoregressive coefficient of the $i$ lag order term of the jump strength $\lambda_{t}, \gamma_{i}$ is the influence coefficient of the jump deviation of the $i$ lag order on the jump strength, and $r, s$ are the lag order. The expectation and deviation of conditional jump strength are:

$$
\lambda_{t} \equiv E\left[n_{t} \mid \Phi_{t-1}\right]
$$




$$
\xi_{t-i} \equiv E\left[n_{t-i} \mid \Phi_{t-i}\right]-\lambda_{t-i}=\sum_{j=0}^{\infty} j P\left(n_{t-i}=j \mid \Phi_{t-i}\right)-\lambda_{t-i}
$$

The ARJI-R2-GARCH model is the Jump-GARCH model in which the mean value of jump range follows the process of conditional autoregression (AR), and the jump variance is related to the variance of realized return (R2). For the ARJI-R2-GARCH model, we assume that the mean and variance of jump amplitude $Y_{t, k} \sim N\left(\theta_{t}, \delta_{t}^{2}\right)$ are conditionally dynamic and related to the return on assets in the previous period:

$$
\begin{gathered}
\theta_{t}=\eta_{0}+\eta_{1} R_{t-1} D\left(R_{t-1}\right)+\eta_{2} R_{t-1}\left(1-D\left(R_{t-1}\right)\right) \\
\delta_{t}^{2}=\zeta_{0}+\zeta_{1} R_{t-1}^{2}
\end{gathered}
$$

where $\left\{\begin{array}{l}D(x)=1, \quad x>0 \\ D(x)=0, \quad \text { else }\end{array} ; \eta_{0}, \eta_{1}, \eta_{2}, \zeta_{0}\right.$, and $\zeta_{1}$ are all parameters to be estimated.

For the ARJI-H-GARCH model, we assume that the variance of jump amplitude is affected by market volatility [40,41], namely:

$$
\delta_{t}^{2}=\zeta_{0}+\zeta_{1} h_{t}
$$

\subsection{Parameter Estimation of Jump-GARCH Model}

The parameter estimation of the jump GARCH model is based on log maximum likelihood estimation. Assume that the conditional probability density function of the return rate $R_{t}$ of assets with $j$ jumps at $t-1$ is $f\left(R_{t} \mid n_{t}=j, \Phi_{t-1}\right)$. At the time of observing $R_{t}$, according to the Bayesian formula, it can be derived that the posterior probability of the occurrence of $j$ jumps at $t$ is as follows:

$$
P\left(n_{t}=j \mid \Phi_{t}\right)=\frac{f\left(R_{t} \mid n_{t}=j, \Phi_{t-1}\right) P\left(n_{t}=j \mid \Phi_{t-1}\right)}{P\left(R_{t} \mid \Phi_{t-1}\right)}, j=0,1,2, \cdots
$$

The conditional density function of the return $R_{t}$ is:

$$
P\left(R_{t} \mid \Phi_{t-1}\right)=\sum_{j=0}^{\infty} f\left(R_{t} \mid n_{t}=j, \Phi_{t-1}\right) P\left(n_{t}=j \mid \Phi_{t-1}\right)
$$

Then the likelihood function and logarithmic likelihood function of the jump GARCH model are expressed as follows:

$$
f\left(R_{t} \mid n_{t}=j, \Phi_{t-1}\right)=\frac{1}{\sqrt{2 \pi\left(h_{t}+j \delta_{t}^{2}\right)}} \times \exp \left[-\frac{\left(R_{t}-\mu-\sum_{i=1}^{l} \phi_{i} R_{t-i}-\theta_{t} j\right)^{2}}{2\left(h_{t}+j \delta_{t}^{2}\right)}\right]
$$

and

$$
L(\Theta)=\ln \left[f\left(R_{t} \mid \Phi_{t-1} ; \Theta\right)\right]
$$

The partial derivative of the above is found and made equal to 0 . Then, the first-order necessary condition equations are achieved, which can be solved to get the estimated values of model parameters.

\subsection{Prediction of Jump Diffusion Volatility}

From the conditional mean value equation in Equations (4) and (5), the formula for calculating the disturbance term can be obtained

$$
\varepsilon_{t}=R_{t}-\mu-\sum_{i=1}^{l} \phi_{i} R_{t-i}=\sqrt{h_{t}} z_{t}+\sum_{k=1}^{n_{t}} Y_{t, k}
$$


It can be seen from Equation (11) that the disturbance term of the jump model includes continuous diffusion volatility component $\varepsilon_{1 t}=\sqrt{h_{t}} z_{t}$ and discrete jump volatility component $\varepsilon_{2 t}=\sum_{k=1}^{n_{t}} Y_{t, k}$, which is caused by significant information impact on the return. In other words, $\varepsilon_{t}=\varepsilon_{1, t}+\varepsilon_{2, t}$. In order to distinguish it from the traditional continuous diffusion volatility, this volatility is called the jump diffusion wave. The jump diffusion volatility $\sigma_{t}^{2}$ of the period return $R_{t}$ based on information set $\Phi_{t-1}$ is:

$$
\begin{aligned}
\sigma_{t}^{2} & =\operatorname{Var}\left(R_{t} \mid \Phi_{t-1}\right)=\operatorname{Var}\left(\varepsilon_{t} \mid \Phi_{t-1}\right) \\
& =h_{t}+\left(\theta_{t}^{2}+\delta_{t}^{2}\right) \lambda_{t}
\end{aligned}
$$

Equation (18) implies that the jump diffusion volatility can be divided into the continuous fluctuation part $h_{t}$ and the jump fluctuation part $\lambda_{t}$.

\subsection{Comparison of the Effectiveness of Jump Diffusion Volatility Prediction}

In order to select the most effective Jump-GARCH model to predict the jump diffusion volatility, we first select the effective model according to the minimum value of final prediction error criterion (FPE), Akaike information criterion (AIC), Bayesian information criterion (BIC), Hannan-Quinn criterion (HQIC), and Schwarz Bayesian information criterion (SBIC). When the AIC criterion and BIC criterion point in different directions, the likelihood ratio test is used, and the model with greater improvement of log likelihood function value is selected as the optimal model.

\subsection{Support Vector Quantile Regression Estimation of VaR}

Multi-period VaR can be estimated from the stock price volatility forecast and single period return based on some complex nonlinear function formula [42], while stock price volatility has the typical characteristics of heavy-tail, clustering, asymmetry, and non-normality. Semi-parametric support vector quantile regression (SVQR) combines the advantages of nonlinear regression and QR estimation. Compared with neural network quantile regression, both in sample and out sample prediction have better estimation effect [43], and its estimation effect is better than parametric methods and linear QRs [15]. Therefore, in order to consider the nonlinear effect of the acceleration mechanism of risk amplification under the impact of abnormal information, we use the SVQR model to estimate the VaR.

The equation of SVQR model is:

$$
Y_{t}=f\left(X_{t}\right)=\mathbf{w}_{\tau}^{\prime} \varphi\left(X_{t}\right)+b_{\tau}+\varepsilon_{t}
$$

where $\mathbf{w}_{\tau}$ represents the parameter vector, $b_{\tau}$ represents the threshold, $\varepsilon_{t}$ represents the disturbance term, $\varphi(\cdot)$ represents the nonlinear mapping, which transforms the low dimensional nonlinear function into the high-dimensional linear function, $\varphi\left(X_{t}\right)^{\prime} \varphi\left(X_{k}\right)=K\left(X_{t}, X_{k}\right)$, and $K\left(x_{t}, x_{k}\right)$ is the kernel function. The optimal parameters $(w, b)$ are obtained by training the sample set $\left\{Y_{t} ; X_{t}\right\}$.

The objective function of SVQR model parameter estimation is defined as follows:

$$
\min _{\mathbf{w}, b} \frac{1}{2} \mathbf{w}^{\prime} \mathbf{w}+\lambda \sum_{t=1}^{T} \rho_{\tau}\left(Y_{t}-\mathbf{w}_{\tau}^{\prime} \varphi\left(X_{t}\right)-b_{\tau}\right)
$$

where $T$ represents the sample size and $\lambda$ represents the penalty parameter.

The Lagrange multiplier is introduced to construct the Lagrange function, and the necessary conditions for solving the first order extreme value are obtained:

$$
\hat{w}_{\tau}=\sum_{t=1}^{T}\left(\alpha_{\tau, t}-\alpha_{\tau, t}^{*}\right) \varphi\left(X_{t}\right)
$$




$$
\hat{b}_{\tau}=\frac{1}{\left|I_{\tau, s v}\right|} \sum_{\tau, t s v \in I_{\tau, s v}}\left(Y_{\tau, t s v}-\sum_{t=1}^{T}\left(\alpha_{\tau, t}-\alpha_{\tau, t}^{*}\right) K\left(X_{t}, X_{\tau, t s v}\right)\right)
$$

where the subscript set of support vector at different quantiles $\tau, t s v \in I_{\tau, s v}$ :

$$
I_{\tau, S v}=\left\{t=1,2, \cdots, T \mid 0<\alpha_{\tau, t}<\tau \lambda, 0<\alpha_{\tau, t}^{*}<(1-\tau) \lambda\right\}
$$

In this paper, we use the kernel function $K\left(x_{t}, x_{k}\right)$ in the form of Gaussian function:

$$
K\left(X_{t}, X_{k}\right)=\exp \left(-\frac{\left\|X_{t}-X_{k}\right\|^{2}}{2 \sigma_{K}^{2}}\right)
$$

The optimal set value of penalty parameter $\lambda$ and variance $\sigma_{K}^{2}$ in radial basis function is determined by the generalized approximate cross validation criterion.

When estimating the VaR of unit assets in each holding period, we use the QR method to solve the problems of nonlinear structure and tail heterogeneity in economic behavior, and the estimation result is more effective than the parameter method. On the other hand, the $Q R$ method assumes that the multi period VaR depends on the square root, the first and second terms of holding period, and the first, second, and higher terms of volatility [42]. When estimating the VaR of assets in each holding period, the VaR mainly depends on the first and second term of volatility [43]. Thus, the first and second term $\hat{\sigma}_{t}, \hat{\sigma}_{t}^{2}$ of conditional volatility are selected as the input variables of the SVQR model. According to the SVQR model, the estimated formula of VaR is as follows:

$$
\operatorname{VaR}_{\tau, t}=\hat{Q}_{Y_{t}}\left(\tau \mid X_{t}\right)=\hat{\mathbf{w}}_{\tau}^{\prime} \varphi\left(\hat{\sigma}_{t}, \hat{\sigma}_{t}^{2}\right)+\hat{b}_{\tau}
$$

\subsection{Validity Test of VaR Estimation}

The SVQR model is a QR of support vector of semiparametric method. In order to verify the effectiveness of the jump GARCH-SVQR model in estimating the VaR of banks, this paper compares the relative effectiveness of the three models by using the Kupiec failure probability test and dynamic quantile test based on parametric $Q R$ and nonparametric $Q R$.

\subsubsection{Parametric Quantile Regression and Nonparametric Quantile Regression}

Following [43], we set the QR model as follows:

$$
V a R_{\tau}=\beta_{0, \tau}+\beta_{1, \tau} \hat{\sigma}_{t}+\beta_{2, \tau} \hat{\sigma}_{t}^{2}+\varepsilon_{t}
$$

where, $\beta_{0, \tau}, \beta_{1, \tau}$, and $\beta_{2, \tau}$ are the parameters to be estimated, $\varepsilon_{t}$ is the disturbance term and $\hat{\sigma}_{t}$ is the conditional volatility estimated by the Jump-GARCH model.

The nonparametric $\mathrm{QR}$ model uses explanatory variables of the parametric $\mathrm{QR}$ model, and the estimates are done by B-spline $\mathrm{QR}$ model.

\subsubsection{Failure Probability Test}

According to the definition of VaR, the failure rate of the estimated VaR should be equal to the significance level. If the actual inspection days are $T$ and the failure days are $N$, then the failure frequency is $p=N / T$. According to the Kupiec failure probability test, the failure frequency obeys the binomial distribution of expected probability $p^{*}$. The original hypothesis is $H_{0}: p=p^{*}$, the likelihood ratio test statistic is constructed:

$$
L R=-2 \ln \left[\left(1-p^{*}\right)^{T-N} \times p^{* N}\right]+2 \ln \left[(1-p)^{T-N} \times p^{N}\right] \sim \chi^{2}(1)
$$


When the $L R$ statistic value $P$ is less than the given signal level, the original model is rejected. On the contrary, when the value $P$ is greater than the critical value, the model is accepted. The higher the value $P$, the better the effect of risk estimation.

\subsubsection{Dynamic Quantile Test}

Dynamic quantile test mainly tests whether there is correlation among the failures of VaR estimations. The test statistics are defined as:

$$
H I T_{\alpha, t}=I\left(r_{t}<-V a R_{t}\right)-\alpha
$$

where $\alpha$ is the given confidence level and $I(\cdot)$ is the indicator function. If $r_{t}<-V a R_{t}, H I T_{\alpha, t}=1-\alpha$. If $r_{t}>-V a R_{t}, H I T_{\alpha, t}=-\alpha$. The auxiliary regression equation is constructed as follows:

$$
H I T_{\alpha, t}=\beta_{0}+\beta_{1} H I T_{\alpha, t-1}+\cdots+\beta_{p} H I T_{\alpha, t-p}+\beta_{p+1} V a R_{\alpha, t}+u_{t}
$$

The above model can be expressed in matric form: $H_{1, t}=\mathbf{X} \boldsymbol{\beta}+u_{t}$, where $\mathbf{X}=$ $\left(1, H_{i t} t_{\alpha, t-1}, \cdots, H i t_{\alpha, t-p}, V a R_{\alpha, t}\right), \beta=\left(\beta_{0}, \beta_{1}, \cdots, \beta_{p}, \beta_{p+1}\right)$, and $u_{t} \sim N\left(0, \sigma_{u}^{2}\right)$.

If the model parameters are estimated correctly, we can get $E\left(H I T_{\alpha, t} \mid \Omega_{t-1}\right)=0$. This shows that there should be no correlation among $H_{I} T_{\alpha, t}, H I T_{\alpha, t-k}$, and those predicted $V a R_{\alpha, t}$. Therefore, the null hypothesis of dynamic quantile test is $\beta_{0}=\beta_{1}=\cdots=\beta_{p+1}=0$. The constructed test statistics $D Q$ are as follows:

$$
D Q=\frac{\hat{\boldsymbol{\beta}}_{\text {ols }}^{\prime} \mathbf{X}^{\prime} \mathbf{X} \hat{\boldsymbol{\beta}}_{\text {ols }}}{\alpha(1-\alpha)} \sim \chi^{2}(p+2)
$$

When the $D Q$ statistic is less than the critical value of chi-square distribution under the given confidence level, the null hypothesis is accepted, and the independence of $V a R$ sequences is confirmed. Otherwise, the model is rejected.

\section{Empirical Results and Further Analysis}

\subsection{Sample Data and Descriptive Statistics}

We consider the banking industry in China and use the data of those banks that are listed on the Chinese stock markets. As a new account rule was introduced in 2007, we select all banks that were publicly traded before the new accounting rule was implemented, so the results can be comparable. It was discovered that there were only 14 commercial banks listed before that. Although the number of banks seems to be small, those 14 banks include banks of big, medium, and small sizes, and they are good representatives for the whole Chinese banking industry. In particular, three of the biggest banks, Industrial and Commercial Bank of China (ICBC), China Construction Bank (CCB), and Bank of China (BOC) are among the 14 banks we consider here. Some other banks, such as Agricultural Bank of China, went public after the new account rule was implemented, and they are excluded from our empirical study here.

In addition, our research mainly focuses on the single variable time series of a single sample, and we do not consider cross-sectional effects. The sample environment and the number of samples should not affect the single sample conclusion and its robustness, and its principle and conclusion should be the same as that of other countries' financial market risk measurement.

The data collection period of this paper is from 25 September 2007, to 15 November 2019. Excluding non-trading dates, a total of 2953 data (daily closing prices, banks equities, etc.) were collected. The indicators were all from the Wind database. We calculate the daily logarithmic rate of return of the whole banking industry by weighted average with the proportion of each bank's current equity in the banking industry as the weight. All data analysis in this paper is implemented by MATLAB software. 
According to the descriptive statistics of the sample data, the average return of each bank is around 0 . The standard deviations of their yields are small, and the fluctuations are stable in general. Most of the bank return data are obviously left-skewed, which implies that the probability of return occurring on the left side of the mean value is greater than that on the right side. The kurtosis values of all bank yield series are large, and the yield series have obvious characteristics of "peak and heavy-tail". JB statistics are significant at the $1 \%$ confidence level, which shows that the series of bank yields do not obey normal distribution. The unit root ADF test shows that all yield series are stationary time series, and there is no pseudo regression phenomenon.

\subsection{Jump Identification and Analysis}

Asset prices in the financial market are vulnerable to the impact of discrete random events, and jump behavior is identified by using a time-varying threshold estimation value. The results are shown in Figure 1. It can be seen from Figure 1 that during 2008, affected by the global financial crisis, market return jumped in April, June, and September, respectively, and jumps occurred from time to time during 2009-2012. Affected by the "money shortage" event in 2013, jumping events occurred in four quarters, and there were three jumps in 2014. During the "stock disaster" of China's A-share market in June 2015 and the stock market circuit breaker in early January 2016, the market return rate showed a significant jump phenomenon. Therefore, it is meaningful to explore financial crisis from the perspective of jump risk. Jump events are often accompanied by obvious volatility clustering. The Jump-GARCH model can be used to describe the clustering characteristics of jump volatility of market return series.

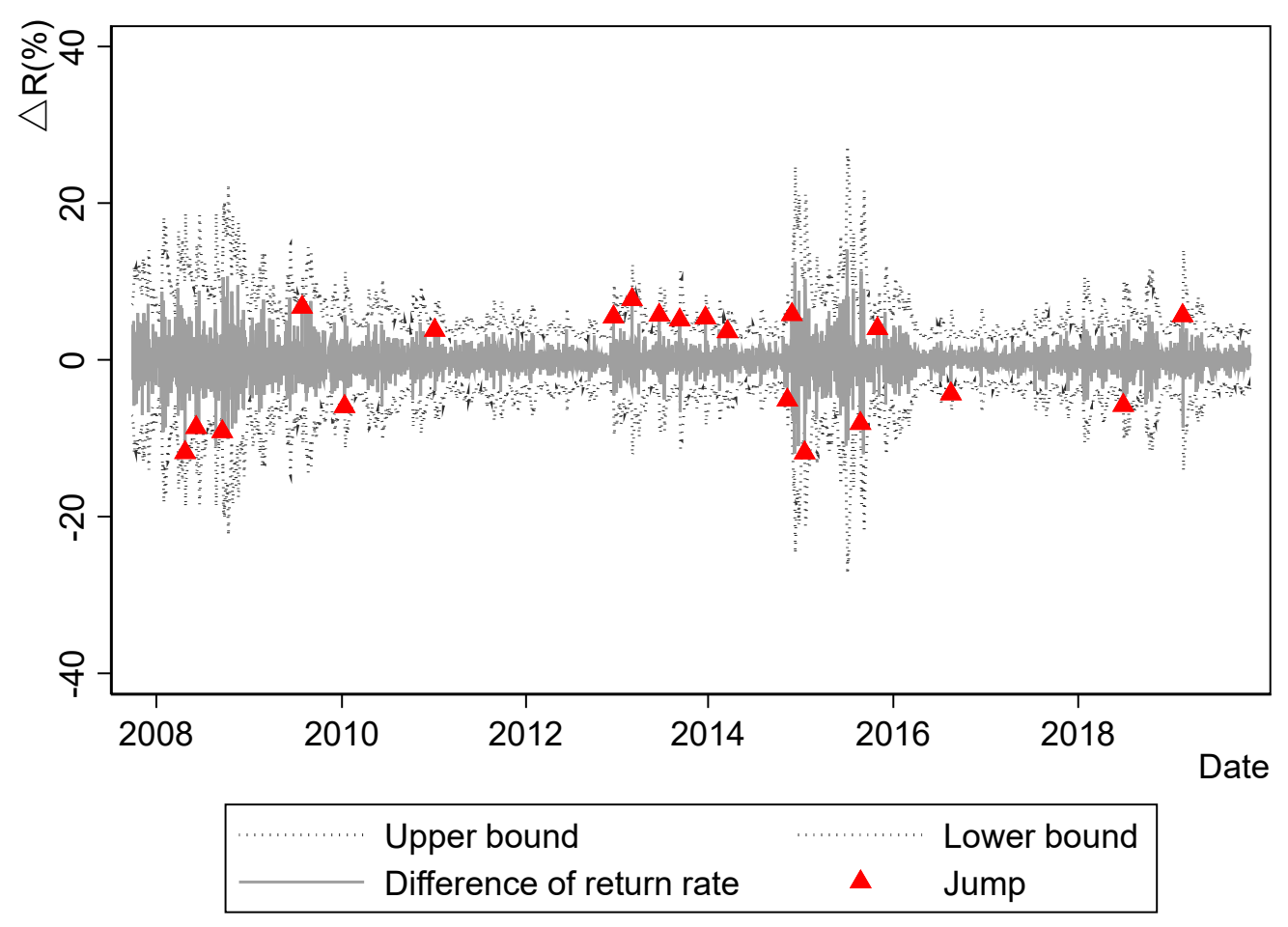

Figure 1. Trend chart of market closing price and yield.

\subsection{Prediction Results and Analysis of Bank Jump Diffusion Volatility}

\subsubsection{The Jump-GARCH Model and Comparison of the Effectiveness of Volatility Prediction}

The information criterion of the autoregressive model with a five order lag rate of return is calculated. The value of FPE, AIC, BIC, HQIC, and SBIC is minimized, and the conditional mean equation only contains constant terms and random terms. 
The LBQ test and ARCH-LM test indicate that there is ARCH effect in yield series, so a GARCH model should be used. According to the principle of information criterion minimization, the $\operatorname{GARCH}(1,1)$ model is selected. We consider the discrete jump component in the mean equation of the model, and we use the maximum likelihood estimation method (12) to estimate the parameters. Four kinds of Jump-GARCH models are constructed for each bank, and the effectiveness of the models is compared and tested.

The results show that most of the models can be improved by adjusting the autocorrelation of disturbance term. In addition, AIC criterion and BIC criterion match well. Among the banks we considered, PingAn Bank has the problem of model validation. The likelihood ratio test $L R(3)$ is 22 , and the critical value corresponding to $95 \%$ confidence level $L R(3)$ is 7.81 . Therefore, the constrained ARCH-GARCH model should be rejected, and the ARJI-R2-GARCH model is considered to be the most suitable model to describe the volatility of PingAn Bank with jump characteristics. Bank of Ningbo also has the problem of model validation. The likelihood ratio test $L R(5)$ is 20 , and the critical value $L R(5)$ corresponding to $95 \%$ confidence level is 11.07 . Therefore, the constrained CJ-GARCH model should be rejected, and the ARJI-R2-GARCH model is considered to be the most suitable model to describe the volatility of Ningbo bank with jump characteristics.

After the effectiveness comparison, the optimal Jump-GARCH models selected by banks are summarized in Table 1.

Table 1. Summary of optimal jump diffusion volatility models of commercial banks.

\begin{tabular}{|c|c|c|}
\hline Model & CJ-GARCH & ARJI-R²-GARCH \\
\hline Bank & $\begin{array}{c}\text { HuaXia Bank Co., Ltd. (HXBC), } \\
\text { China Minsheng Banking Corp., Ltd. (CMBC), } \\
\text { China Merchants Bank Co., Ltd. (CMB), } \\
\text { Bank of Nanjing Co., Ltd. (BNC), } \\
\text { Industrial Bank Co., Ltd., (CIB), } \\
\text { Bank of Communications (BCM), } \\
\text { Industrial and Commercial Bank of China (ICBC), } \\
\text { Bank of China (BOC), } \\
\text { China CITIC Bank (CITIC) }\end{array}$ & $\begin{array}{c}\text { PingAn Bank (PAB) } \\
\text { Bank of Ningbo (BON) } \\
\text { Shanghai Pudong Development } \\
\text { Bank (SPDB) } \\
\text { Bank of Beijing(BOB) } \\
\text { China Construction Bank (CCB) }\end{array}$ \\
\hline \multicolumn{3}{|c|}{$\begin{array}{l}\text { China's commercial banks are more suitable for CJ-GARCH models with constant jump intensity and } \\
\text { ARJI-R2-GARCH models with autoregressive characteristics and dynamic jump range. Among them, } \\
\text { the ARJI-R2-GARCH model reflects that conditional mean and conditional variance of jump range are sensitive to } \\
\text { yields, which can be used to describe the effect of direction change and size change on jump characteristics. }\end{array}$} \\
\hline
\end{tabular}

\subsubsection{Statistics of Jump Diffusion Volatility Forecast Value of Commercial Banks}

By virtue of the optimal Jump-GARCH model of banks in Table 1 and Formula (17), we get the jump diffusion volatility of 14 commercial banks, and the statistical results are shown in Table 2.

It can be seen from Table 2 that the relative amplitude (dispersion coefficient) of each bank's jump diffusion fluctuation is all around 1, and the main difference lies in the absolute amplitude of their jump diffusion volatility. Bank of China, Industrial and Commercial Bank of China, China Construction Bank, and Bank of Communications, are the four big state-owned banks and. their jump diffusion volatility mean and standard deviation are small, far lower than the overall average level, which shows that the stock price of big state-owned banks has strong anti-shock ability, and their self-recovery ability after the impact is also good. On the other hand, PingAn Bank, Ningbo Bank, Shanghai Pudong Development Bank, Huaxia Bank, Bank of Nanjing, Industrial Bank, Bank of Beijing, and China CITIC Bank all have higher means and standard deviations than the average. Among the eight banks mentioned above, PingAn Bank, Shanghai Pudong Development Bank, and Bank of Nanjing are the top three volatile banks, which indicates that under the extreme financial impact, their jump volatility is more severe and their stocks are vulnerable. 
Table 2. Descriptive statistics of jump diffusion volatility of 14 commercial banks.

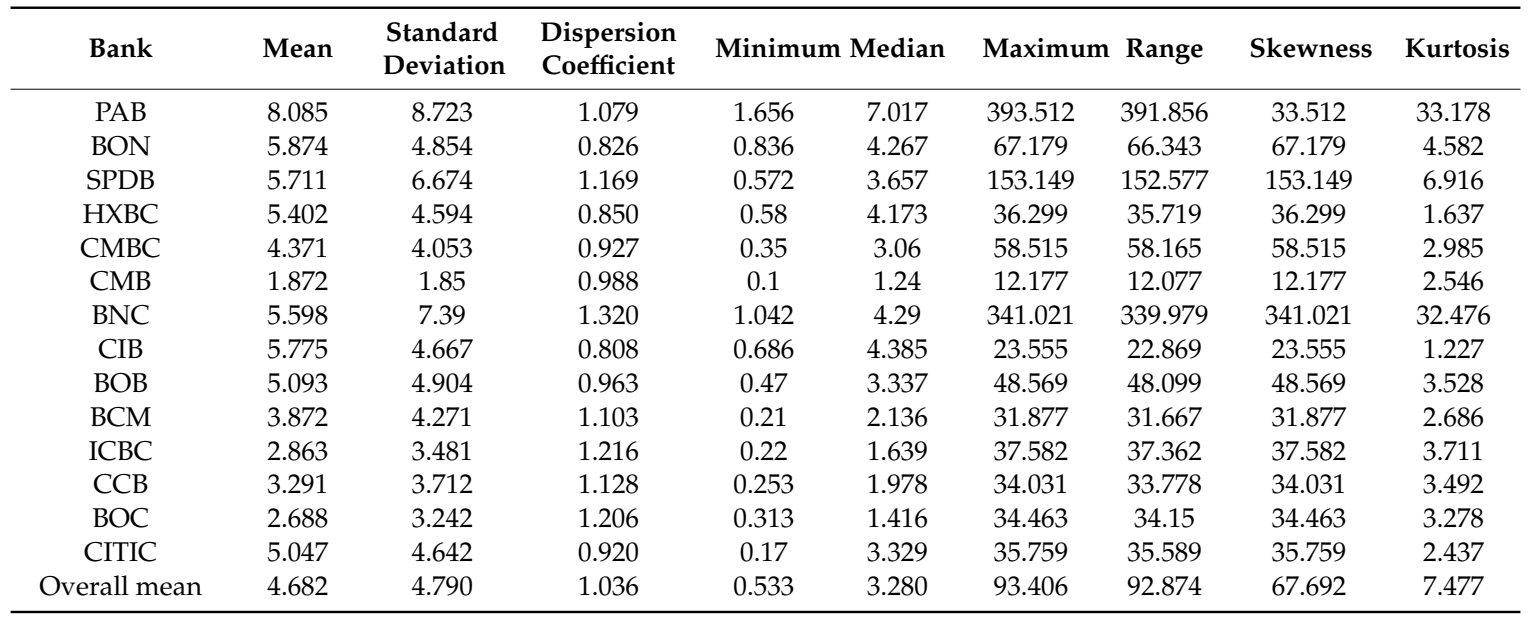

The conditional volatility distribution of all banks is significantly right-skewed, peak, and heavy-tail, which indicates that the jump diffusion caused by the impact of extreme risk events makes the VaR have many extreme deviations. Risk management teams should pay special attention to these extreme financial events that will lead to a surge in risk.

The HAR-type models focus on the long memory of jumps, and use the autoregressive technology to predict the volatility of financial asset returns with high accuracy $[15,44,45]$. However, the HAR-type models cannot handle the significant characteristics of financial asset return volatility, such as heavy tail, right deviation, clustering, and leverage effect. Differently from the HAR-type model, the Jump-GARCH model takes into account the continuous jump diffusion characteristics at the same time, systematically predicts the volatility, and gives the wave characteristic parameters comprehensively.

\subsection{SVQR Estimation and Analysis of Banks' Jump Diffusion VaR}

Firstly, by virtue of the generalized approximate cross validation method, we have 16 parameter combinations by the combination of $\lambda=[100,500,1000,1500]$ and $\sigma_{K}^{2}=[1,5,10,15]$. The optimal combination of parameters is selected by minimizing $\operatorname{GACV}\left(\lambda, \sigma_{K}^{2}\right)$. Then, the optimal parameter combination and the Equation (27) are used to calculate the estimated parameters of the SVQR model. The jump diffusion volatility prediction value and formula (28) are used to calculate the jump diffusion VaR prediction sequence of each bank, and the descriptive statistical information is shown in Table 3 .

Table 3. Descriptive statistics of VaR of commercial banks.

\begin{tabular}{|c|c|c|c|c|c|c|c|c|c|}
\hline Bank & Mean & $\begin{array}{l}\text { Standard } \\
\text { Deviation }\end{array}$ & $\begin{array}{c}\text { Dispersion } \\
\text { Coeff. }\end{array}$ & Minimum & Median & Maximum & Range & Skewness & Kurtosis \\
\hline PAB & -3.687 & 1.318 & -0.357 & -18.1 & -3.26 & 2.202 & 20.302 & -4.314 & 32.164 \\
\hline SPDB & -3.354 & 1.593 & -0.475 & -7.78 & -3.087 & -0.576 & 7.204 & -0.589 & 2.559 \\
\hline HXBC & -3.489 & 1.79 & -0.513 & -12.029 & -3.239 & -0.901 & 11.128 & -0.911 & 3.492 \\
\hline CMBC & -2.881 & 1.263 & -0.438 & -10.718 & -2.642 & -0.801 & 9.917 & -0.879 & 4.024 \\
\hline CIB & -3.415 & 1.832 & -0.536 & -9.498 & -3.026 & -1.008 & 8.49 & -0.865 & 3.092 \\
\hline BOB & -3.047 & 1.388 & -0.456 & -6.036 & -2.71 & 0.96 & 6.996 & -0.581 & 3.200 \\
\hline BCM & -2.745 & 1.488 & -0.542 & -8.309 & -2.231 & -0.115 & 8.194 & -1.202 & 3.931 \\
\hline $\mathrm{ICBC}$ & -2.23 & 1.059 & -0.475 & -6.346 & -1.943 & -0.49 & 5.856 & -1.152 & 3.954 \\
\hline $\mathrm{CCB}$ & -2.553 & 1.364 & -0.534 & -9.06 & -2.131 & -0.262 & 8.798 & -1.586 & 5.794 \\
\hline
\end{tabular}


It can be seen from Table 3 that the overall average value of jump diffusion VaR of all banks is 3.07. The average level of jump diffusion VaR of state-owned banks is relatively low, and the range of deviation is relatively small, which further indicates that they have better shock resistance. On the other hand, the joint-equity commercial banks and urban development banks, except Minsheng Bank, have higher jump diffusion risk average level and higher deviation range. Among them, PingAn Bank, Huaxia Bank, Minsheng Bank, and Bank of Nanjing have greater changes than the overall average. PingAn Bank is the largest, which is about twice the overall average, indicating that it is relatively fragile and its resilience to shocks and self-recovery ability is relatively low.

The skewness of VaR of each bank is near 0 and slightly towards the left, and the kurtosis fluctuates around 3, which indicates that the estimated jump VaR distribution has the characteristics of peak and heavy-tail. It also implies that the extreme VaR value caused by jump diffusion is significant. Among them, the maximum and kurtosis of jump risk value of PingAn Bank are outliers, and the effectiveness analysis should be conducted.

\subsection{Effectiveness Test Results and Analysis of VaR Estimation of Bank Jump Diffusion}

In order to check the validity of Jump-GARCH-SVQR model, two similar techniques, parametric $\mathrm{QR}$ and nonparametric $\mathrm{QR}$, are used to estimate the VaR. The failure probability test and dynamic quantile test are used to compare the estimation effect of the three methods. The results are shown in Table 4.

Table 4. Validity test of VaR estimation results.

\begin{tabular}{|c|c|c|c|c|c|c|}
\hline \multirow{2}{*}{ Bank } & \multicolumn{3}{|c|}{ Failure Probability Test (LR) } & \multicolumn{3}{|c|}{ Dynamic Quantile Test (DQ) } \\
\hline & QR & NQR & SVQR & QR & NQR & SVQR \\
\hline \multirow{2}{*}{ PAB } & 0.014 & 0.014 & 0.014 & 6.880 & 10.515 & 7.299 \\
\hline & [0.906] & [0.906] & [0.906] & [0.230] & [0.062] & [0.199] \\
\hline \multirow{2}{*}{$\mathrm{BON}$} & 0.082 & 0.041 & 0.014 & 3.577 & 2.097 & 2.337 \\
\hline & [0.775] & [0.840] & [0.906] & [0.612] & [0.836] & [0.801] \\
\hline \multirow{2}{*}{ SPDB } & 0.041 & 0.041 & 0.014 & 2.510 & 1.029 & 0.749 \\
\hline & {$[0.840]$} & {$[0.840]$} & [0.906] & [0.775] & [0.960] & [0.980] \\
\hline \multirow{2}{*}{$\mathrm{HXBC}$} & 0.041 & 0.014 & 0.014 & 6.706 & 7.018 & 6.345 \\
\hline & {$[0.840]$} & [0.906] & [0.906] & {$[0.244]$} & [0.219] & {$[0.274]$} \\
\hline \multirow{2}{*}{ CMBC } & 0.082 & 0.041 & 0.001 & 9.974 & 8.000 & 7.108 \\
\hline & [0.775] & [0.840] & [0.973] & [0.076] & [0.156] & [0.213] \\
\hline \multirow{2}{*}{ CMB } & 0.041 & 0.041 & 0.014 & 2.015 & 2.056 & 3.053 \\
\hline & [0.840] & [0.840] & [0.906] & [0.847] & [0.841] & [0.692] \\
\hline \multirow{2}{*}{$\mathrm{BNC}$} & 0.041 & 0.041 & 0.014 & 2.929 & 3.004 & 1.329 \\
\hline & {$[0.840]$} & [0.840] & [0.906] & [0.711] & [0.699] & [0.932] \\
\hline \multirow{2}{*}{ CIB } & 0.014 & 0.041 & 0.041 & 1.309 & 0.967 & 1.769 \\
\hline & [0.906] & [0.840] & {$[0.840]$} & [0.934] & [0.965] & [0.880] \\
\hline \multirow{2}{*}{ BOB } & 0.082 & 0.041 & 0.014 & 2.742 & 2.786 & 2.822 \\
\hline & [0.775] & [0.840] & [0.906] & [0.740] & [0.733] & [0.727] \\
\hline \multirow{2}{*}{ BCM } & 0.041 & 0.014 & 0.014 & 0.749 & 1.322 & 1.043 \\
\hline & [0.840] & [0.906] & [0.906] & [0.980] & [0.933] & [0.959] \\
\hline \multirow{2}{*}{ ICBC } & 0.041 & 0.041 & 0.014 & 0.275 & 0.677 & 0.426 \\
\hline & [0.840] & [0.840] & [0.906] & [0.998] & [0.984] & [0.995] \\
\hline \multirow{2}{*}{ ССВ } & 0.082 & 0.041 & 0.014 & 3.347 & 2.967 & 3.475 \\
\hline & [0.775] & [0.840] & [0.906] & [0.647] & [0.705] & [0.627] \\
\hline
\end{tabular}


Table 4. Cont.

\begin{tabular}{ccccccc}
\hline \multirow{2}{*}{ Bank } & \multicolumn{2}{c}{ Failure Probability Test (LR) } & \multicolumn{3}{c}{ Dynamic Quantile Test (DQ) } \\
\cline { 2 - 7 } & QR & NQR & SVQR & QR & NQR & SVQR \\
\hline \multirow{2}{*}{ BOC } & 0.041 & 0.014 & 0.014 & 1.272 & 1.309 & 0.142 \\
& {$[0.840]$} & {$[0.906]$} & {$[0.906]$} & {$[0.938]$} & {$[0.934]$} & {$[1.000]$} \\
\hline \multirow{2}{*}{ CITIC } & 0.041 & 0.014 & 0.014 & 7.139 & 5.088 & 3.876 \\
& {$[0.840]$} & {$[0.906]$} & {$[0.906]$} & {$[0.211]$} & {$[0.405]$} & {$[0.567]$} \\
\hline Model average error & $0.086 \%$ & $0.069 \%$ & $0.047 \%$ & & & \\
\hline
\end{tabular}

Note: (1) In "[]" is the probability $p$ value of the inspection quantity, and the upper part is the inspection statistics; (2) "Model average error" refers to the average level of deviation between failure probability and expected probability. (3) "QR", "NQR", and "SVQR" refer to parametric quantile regression, nonparametric quantile regression, and support vector quantile regression, respectively.

It can be seen from Table 4 that all the 14 banks have passed the test in the two posterior tests of SVQR model for estimating jump VaR. The dynamic quantile test fails in the parametric QR model and nonparametric $\mathrm{QR}$ model in banks' VaR estimation. For example, when the parametric model is used to estimate the VaR of Minsheng Bank and the nonparametric model is used to estimate the VaR of PingAn Bank, both of them have not passed the test. Sequence correlation was found in all cases. Compared with the parametric QR model and nonparametric QR model, the Jump-GARCH-SVQR model has the lowest prediction error. Combining the two tests, the Jump-GARCH-SVQR model is more applicable, and the effect is more robust and accurate.

The reason is that, compared with the Linear QR [46], the Jump-GARCH-SVQR model can describe the change process characteristics of risk factors, and especially can reveal the jumping activity law under the impact of extreme risk events. Moreover, the Jump-GARCH-SVQR model does not need a specific structure. Therefore, considering the nonlinear effect of risk factors, it can reduce the model error and improve the accuracy of VaR prediction, compared to the linear QR model [47]. Even if the empirical distribution was used to estimate the VaR without setting the model structure, which partially reduced the error of model setting [48], it still only considers the linear effect of risk factors. Unlike the linear QR model, the Jump-GARCH-SVQR model not only considers the non-linear effect of risk factors, but also characterizes the complex amplification acceleration mechanism of risk factors caused by risk factors.

\section{Summary and Conclusions}

"Black swan" crises, such as extreme financial events caused by abnormal information shock, often occur in stock markets. The existing literature mostly estimates the early-warning risk based on the continuous diffusion GARCH process model, which is difficult to use to describe and analyze the risk amplification acceleration mechanism of price jump clustering during the crisis. In addition, it cannot accommodate the nonlinear action mechanism and risk mutation of bank risk factors.

In this paper, we use four Jump-GARCH models to predict the time-varying conditional volatility of bank stock price return by using the five information criterion values of FPE, AIC, BIC, HQIC, and SBIC. In addition, the Jump-GARCH model with the best prediction effect is selected for each bank, and the optimal Jump-GARCH model is used to predict the jump diffusion volatility of the stocks. SVQR combines the advantages of nonlinear regression and QR estimation. In order to describe the asymmetric nonlinear mechanism caused by the acceleration mechanism of risk amplification under the abnormal impact of risk factors, we take the first and second term of conditional jump diffusion volatility prediction as the input variable and select SVQR to estimate the VaR. Jump-GARCH-SVQR VaR technology is used to monitor and predict extreme financial events. By using the failure probability test and dynamic quantile test, the validity of VaR estimation of parametric $Q R$, nonparametric $Q R$ and SVQR are verified and compared with each other.

In summary, the empirical study results given the following conclusions: 
(1) The Jump-GARCH model has a better prediction effect than the continuous GARCH model, which indicates that the bank stock price process has a continuous Brownian motion component, discrete jump component, and random volatility component.

(2) Generally speaking, the volatility series of China's listed banks have a leverage effect, long memory effect, and clustering effect, which are mainly applicable to the CJ-GARCH model and ARJI-R2-GARCH model. However, the heterogeneity of the jump process characteristics of each bank is significant, and different sample banks may have different jump models. In the CJ-GARCH model, the mean and variance of jump times and jump amplitude are constant. It was discovered that CJ-GARCH models are suitable for most of the banks we consider. The jump intensity of the ARJI-R2-GARCH model has conditional autoregressive characteristics, and its jump amplitude is dynamic correlation and asymmetric, which is mainly applicable to small and medium-sized banks.

(3) Compared with the results of parametric $Q R$ and nonparametric $Q R$, the risk measurement method of the Jump-GARCH model combined with the SVQR model does not need the hypothesis of data distribution characteristics, and it has the comparative advantage of comprehensive fitting linear and nonlinear effects. The results show that the prediction effect of banks' VaR is more robust and accurate.

Some future research can be conducted to extend the results in this paper. The Jump-GARCH model, combined with the SVQR model, has obvious comparative advantages in measuring market risk and flexible structure framework, which provides a development basis for further research. One future research topic is the diversification of risk factors. The frequency of their time series can be divided into high and low frequency, such as daily financial market indicators, monthly macroeconomic financial indicators, and quarterly financial indicators. In order to make full use of information, we can use mixed frequency data sampling technology (MIDAS) to construct a MIDAS Jump-GARCH model to predict volatility and construct unlimited MIDAS-SVQR models. Another idea is to use a modified Z-statistic based on modified realized threshold multi power variation (C_TZ) proposed by Reference [19]. The proposed C_TZ statistics significantly improve the technical efficiency of the jump test estimation. As the realized volatility has the characteristics of long memory, we can use C_TZ statistics to estimate continuous and jump components, autoregressive moving average (ARMA) model for high frequency data. Further, we can use mixing data sampling technology (MIDAS-HARMA) to predict volatility, and then use the generalized random forest quantile regression model (RFQR) proposed in [49] to estimate VaR.

Author Contributions: Conceptualization and methodology: Z.W. and T.P.; software: Q.Z. and M.Z.; validation: Q.Z., M.Z. and Z.W.; formal analysis: Q.Z. and M.Z.; investigation, resources, data curation, and visualization: Q.Z.; writing—original draft preparation: Z.W. and T.P.; writing-review and editing: Z.W. and T.P.; supervision, project administration, and funding acquisition: Z.W. All authors have read and agreed to the published version of the manuscript.

Funding: This research was funded by general project of National Natural Science Foundation of China (NSFC): Research on multidimensional and multiple contagion of banking systemic financial risk in structural change, grant number 71973098 .

Conflicts of Interest: The authors declare no conflict of interest.

\section{References}

1. Brandao-Marques, L.; Correa, R.; Sapriza, H. Government support, regulation, and risk taking in the banking sector. J. Bank. Financ. 2018, 112, 105284. [CrossRef]

2. Occhino, F. Debt-overhang banking crises: Detecting and preventing systemic risk. J. Financ. Stab. 2017, 30, 192-208. [CrossRef]

3. Gutiérrez-López, C.; Abad-González, J. Sustainability in the Banking Sector: A Predictive Model for the European Banking Union in the Aftermath of the Financial Crisis. Sustainability 2020, 12, 2566. [CrossRef] 
4. Moch, N. The Contribution of Large Banking Institutions to Systemic Risk: What Do We Know? A Literature Review. Rev. Econ. 2018, 69, 231-257. [CrossRef]

5. Sánchez García, J.; Cruz Rambaud, S. A GARCH approach to model short-term interest rates: Evidence from Spanish economy. Int. J. Financ. Econ. 2020. [CrossRef]

6. Lovreta, L.; Joaquín López, P. Structural breaks in the interaction between bank and sovereign default risk. SERIEs 2020, 1-29. [CrossRef]

7. Zhou, C. The term structure of credit spreads with jump risk. J. Bank. Financ. 2001, 25, 2015-2040. [CrossRef]

8. Tang, W.; Su, F. Analysis of the impact of extreme financial events on systemic risk: A case study of China's banking sector. Econ. Res. 2017, 52, 17-33.

9. Fulvio, C.; Davide, P.; Roberto, R. Threshold bi-power variation and the impact of jumps on volatility forecasting. J. Econom. 2010, 159, 276-288.

10. Zhao, H. Jump and leverage effect of China's stock market: Based on the realized range variance. Financ. Res. 2012, 11, 179-192.

11. Yang, K.; Chen, L. Analysis on the characteristics of high frequency volatility jump in China's stock market. J. Syst. Eng. 2012, 27, 492-497.

12. Yang, K.; Tian, F.; Lin, H. Estimation of jump, prediction of stock market volatility and evaluation of prediction accuracy. China Manag. Sci. 2013, 21, 50-60.

13. Luo, J.; Chen, L. Realized volatility forecast with the Bayesian random compressed multivariate HAR model. Int. J. Forecast. 2020, 36, 781-799. [CrossRef]

14. Chen, F.; Jin, Q.; Hu, Z. Verification and application of price jump behavior in China's stock market. Financ. Trade Econ. 2018, 39, 74-88.

15. Shim, J.; Kim, Y.; Lee, J.; Hwang, C. Estimating VaR with semiparametric support vector quantile regression. Comput. Stat. 2012, .27, 685-700. [CrossRef]

16. Xu, Q.; Zhang, J.; Jiang, C. Multi period VaR Risk Measurement Based on nonlinear quantile regression model. China Manag. Sci. 2015, 23, 56-65.

17. Liu, Q.; Yao, Q.; Zhao, G. Model averaging estimation for conditional volatility models with an application to stock market volatility forecast. J. Forecast. 2020, 39, 841-863. [CrossRef]

18. Bhowmik, R.; Wang, S.Y. Stock Market Volatility and Return Analysis: A Systematic Literature Review. Entropy 2020, 22, 522. [CrossRef]

19. Corsi, F. A simple approximate long-memory model of realized volatility. J. Financ. Econom. 2008, 7, $174-196$. [CrossRef]

20. Todorova, N. The course of realized volatility in the LME non-ferrous metal market. Econ. Model. 2015, 51, 1-12. [CrossRef]

21. Huang, Z.; Liu, H.; Wang, T. Modeling long memory volatility using realized measures of volatility: A realized HAR GARCH model. Econ. Model. 2016, 52, 812-821. [CrossRef]

22. O'Brien, J.; Paweł, J. An evaluation of bank measures for market risk before, during and after the financial crisis. J. Bank. Financ. 2017, 80, 215-234. [CrossRef]

23. Orhan, M.; Köksal, B. A comparison of GARCH models for VaR estimation. Expert Syst. Appl. 2012, 39, 3582-3592. [CrossRef]

24. Ma, X.M.; Yang, R.X.; Zou, D.; Liu, R. Measuring extreme risk of sustainable financial system using GJR-GARCH model trading data-based. Int. J. Inf. Manag. 2020, 50, 526-537. [CrossRef]

25. Uylangco, K.; Li, S.Q.W. An evaluation of the effectiveness of Value-at-Risk (VaR) models for Australian banks under Basel III. Aust. J. Manag. 2016, 41, 699-718. [CrossRef]

26. Patton, A.J.; Ziegel, J.F.; Chen, R. Dynamic semiparametric models for expected shortfall (and Value-at-Risk). J. Econom. 2019, 211, 388-413. [CrossRef]

27. Wang, C.S.; Zhao, Z.B. Conditional Value-at-Risk: Semiparametric estimation and inference. J. Econom. 2016, 195, 86-103. [CrossRef]

28. Engle, R.F.; Manganelli, S. CAViaR. J. Bus. Econ. Stat. 2004, 22, 367-381. [CrossRef]

29. Taylor, J.W. A quantile regression neural network approach to estimating the conditional density of multiperiod returns. J. Forecast. 2000, 19, 299-311. [CrossRef]

30. Mancini, C. Estimation of the characteristics of the jumps of a general Poisson-diffusion model. Scand. Actuar. J. 2004, 2004, 42-52. [CrossRef] 
31. Tan, Z.; Hu, H. Nonparametric threshold estimation of short-term interest rate jump diffusion model. China Manag. Sci. 2012, 20, 8-15.

32. Poon, S.H.; Granger, C.W. Forecasting volatility in financial markets: A review. J. Econ. Lit. 2003, 41, 478-539. [CrossRef]

33. Asai, M.; Chang, C.L.; Mcaleer, M. Realized stochastic volatility with general asymmetry and long memory. J. Econom. 2017, 199, 202-212. [CrossRef]

34. Chen, L.; Yang, K. Characteristics, prediction models and prediction accuracy comparison of high frequency volatility in China stock market. Syst. Eng. Theory Pract. 2013, 33, 296-307.

35. Wang, J.; Jiang, Y.; Zhu, Y. Prediction of realized volatility based on Realized-GARCH-Kernel Model: The comparison of CHINA and US. Econ. Model. 2020, 91, 428-444. [CrossRef]

36. Fang, T.; Lee, T.H.; Su, Z. Predicting the long-term stock market volatility: A GARCH-MIDAS model with variable selection. J. Empir. Financ. 2020, 58, 36-49. [CrossRef]

37. Yu, X.; Wang, X. Volatility prediction and VaR measurement based on realized GARCH model based on mixing. Stat. Res. 2018, 35, 104-116.

38. Yao, X.; Izzeldin, M.; Li, Z. A novel cluster HAR-type model for forecasting realized volatility. Int. J. Forecast. 2019, 35, 1318-1331. [CrossRef]

39. Chang, K. The time-varying and asymmetric dependence between crude oil spot and futures markets: Evidence from the mixture copula-based ARJI-GARCH model. Econ. Model. 2012, 29, 2298-2309. [CrossRef]

40. Guo, W.; Deng, M.; Dong, Q. Jump characteristics of China's stock market under major events. Syst. Eng. Theory Pract. 2013, 33, 308-316.

41. Taylor, J.W. A quantile regression approach to estimating the distribution of multi-period returns. J. Deriv. 1999, 7, 64-78. [CrossRef]

42. Xu, Q.; Zhang, J.; Jiang, C. Multi period VaR measurement based on support vector quantile regression. J. Syst. Eng. 2014, 29, 202-214.

43. Liu, T.; Zhao, Y. Research on risk measurement of return rate of Shanghai and Shenzhen index based on QR-T-GARCH $(1,1)$ model. Math. Stat. Manag. 2018, 27, 533-543.

44. Choi, K.; Yu, W.C.; Zivot, E. Long memory versus structural breaks in modeling and forecasting realized volatility. J. Int. Money Financ. 2010, 29, 857-875. [CrossRef]

45. Chen, S.L.; Tao, G.; Li, Y.J. Volatility Prediction of stock index futures based on jump, good and bad volatility and Baidu Index. Syst. Eng. Theory Pract. 2018, 38, 299-316.

46. Xu, G.; Lin, B.Q. Jump risk, structural mutation and prediction of crude oil futures price fluctuation. China Manag. Sci. 2018, 26, 11-21.

47. Verma, R.; Ahmad, W.; Uddin, S.G.; Bekiros, S. Analysing the systemic risk of Indian banks. Econ. Lett. 2019, 176, 103-108. [CrossRef]

48. Julia, S. Predicting extreme value at risk: Nonparametric quantile regression with refinements from extreme value theory. Comput. Stat. Data Anal. 2012, 56, 4081-4096.

49. Chen, J.-E.; Hsiang, C.-W. Causal Random Forests Model Using Instrumental Variable Quantile Regression. Econometrics 2019, 7, 49. [CrossRef]

Publisher's Note: MDPI stays neutral with regard to jurisdictional claims in published maps and institutional affiliations.

(C) 2020 by the authors. Licensee MDPI, Basel, Switzerland. This article is an open access article distributed under the terms and conditions of the Creative Commons Attribution (CC BY) license (http://creativecommons.org/licenses/by/4.0/). 